\title{
Simulation of Damage Scenarios in a Bituminous Pavement Tested under FABAC ALT using M4-5n
}

\author{
Olivier Chupin, Jean-Michel Piau, Armelle Chabot, Hanan Nasser, Mai Lan \\ Nguyen
}

LAMES-MAST, IFSTTAR, CS5004, 44344 Bouguenais Cedex, France

olivier.chupin@ifsttar.fr

Yann Lefeuvre

COLAS, Campus scientifique et technique, 4 rue Jean Mermoz, 78772 Magny-les-Hameaux, France

\begin{abstract}
This paper presents the use of a specific layer-wise approach to analyze the results of a FABAC accelerated loading test (ALT) carried out on a flexible pavement with an initial flaw. The approach relies on M4-5n (Multi-particular Model of Multilayer Materials) initially developed for the analysis of debonding in composite materials. M4-5n was recently implemented into a mixed finite element code. This model associated to a Winkler foundation makes it possible to address quite easily problems related to pavements with discontinuities in $3 \mathrm{D}$. The experimental pavement is composed of two bituminous layers and includes initial flaws (metal angles) intended to localize crack growth under repeated moving loads. The pavement is monitored using strain gages and temperature sensors. Moreover, a few FWD campaigns are performed at some steps of the test. The simulations are used to infer the (unknown) pattern of cracking/debonding developing during the first stage of the ALT. Among many patterns tested, one of them is more likely to reflect the experimental facts and is presented hereafter.
\end{abstract}

Keywords M4-5n, debonding, cracking, FABAC device.

This is a pre-print of a contribution published in Accelerated Pavement Testing to Transport Infrastructure Innovation - Proceedings of the 6th APT Conference. In: Chabot A., Hornych P., Harvey J., Loria-Salazar L. (eds) Accelerated Pavement Testing to Transport Infrastructure Innovation, Lecture Notes for Civil Engineering, vol. 96: 389-398. Springer, Cham.

The final authenticated version is available online at: https://doi.org/10.1007/9783-030-55236-7_40 


\section{Introduction}

Associated to different soil modeling, the Multi-particular Model of Multilayer Material (M4) with 5n cinematic fields ( $\mathrm{n}$ : total number of layers), known as M45n (Chabot 1997), was previously proposed (Chabot et al. 2007) (Nasser and Chabot, 2018) as an alternative to other approaches dealing with the modeling of cracks and debonding in pavements (Buttlar et al. 2018). The main advantages of this model are to reduce the actual dimension of the considered problem by one in the solving process and to facilitate the geometrical description of discontinuity areas. It also avoids stress singularities at debonding or crack edges between two materials. The mixed formulation of M4-5n was recently developed and implemented in a finite element code using the FreeFem++ environment (Hecht, 2012) (Nasser et al. 2018). Here, this numerical tool, named M4-FEM, is applied to the $3 \mathrm{D}$ analysis of a full scale accelerated loading test (ALT) conducted on a bituminous pavement using the FABAC device at IFSTTAR. In particular, it is used to investigate cracking/debonding scenarios that possibly developed during the fatigue test.

\section{The FABAC ALT Test}

The FABAC machines used in this study were initially designed and built to test rigid pavements (Aunis and Balay 1998). Since then these linear traffic simulators have been used at IFSTTAR for many other types of experiments such as bond fatigue life of composite (concrete over asphalt) pavements (Chabot et al., 2008). The FABAC machines are equipped with four dual tires placed along a chain in close loop (Figure 1). The length of the loaded section is $2 \mathrm{~m}$ with only one semiaxle applied at a time (maximum load of $75 \mathrm{kN}$ ). The loads move at a maximum speed of $7 \mathrm{~km} / \mathrm{h}$ without lateral wandering.

The main experimental facts and findings of the present ALT test are summarized below. A detailed description of the experiment is given in (Nguyen et al. 2019).

The pavement structure is composed of a $6 \mathrm{~cm}$-thick layer of high modulus asphalt material (HMAM) covered by a $5 \mathrm{~cm}$-thick base course of asphalt material (BC) (Figure 1). A tack coat with $300 \mathrm{~g} / \mathrm{m}^{2}$ residual bitumen is applied at the interface. The asphalt layers rest on an unbound graded aggregate subbase (UGA) of thickness $33 \mathrm{~cm}$ built on the natural soil. Two metal angles are placed at the bottom of the HMAM layer along the transversal direction and at mid-length of the circulated area. These flaws aim at initiating and localizing crack growth in the pavement. The structure is instrumented by means of temperature sensors (at $z=0,5$ and $11 \mathrm{~cm}$ from the pavement surface), longitudinal strain gages at the bottom of layers 1 and 2 respectively and vertical strain gages at top of the UGA 
layer (see Figure 1 for the gage locations). The horizontal gages are located below the right wheel-path and the vertical ones in the longitudinal mid-plane. In addition, FWD campaigns were planned at some steps. These were carried out by moving the FWD plate every $10 \mathrm{~cm}$ along the longitudinal axis running between the dual wheel paths of the FABAC machine.

About 1.5 million of FABAC loads of $65 \mathrm{kN}$ (also named load cycles) were applied in total at a speed equal to $3.7 \mathrm{~km} / \mathrm{h}$. In the following, the analysis of the experimental data is limited to the first 350,000 cycles which already show important changes in the behavior of the structure.

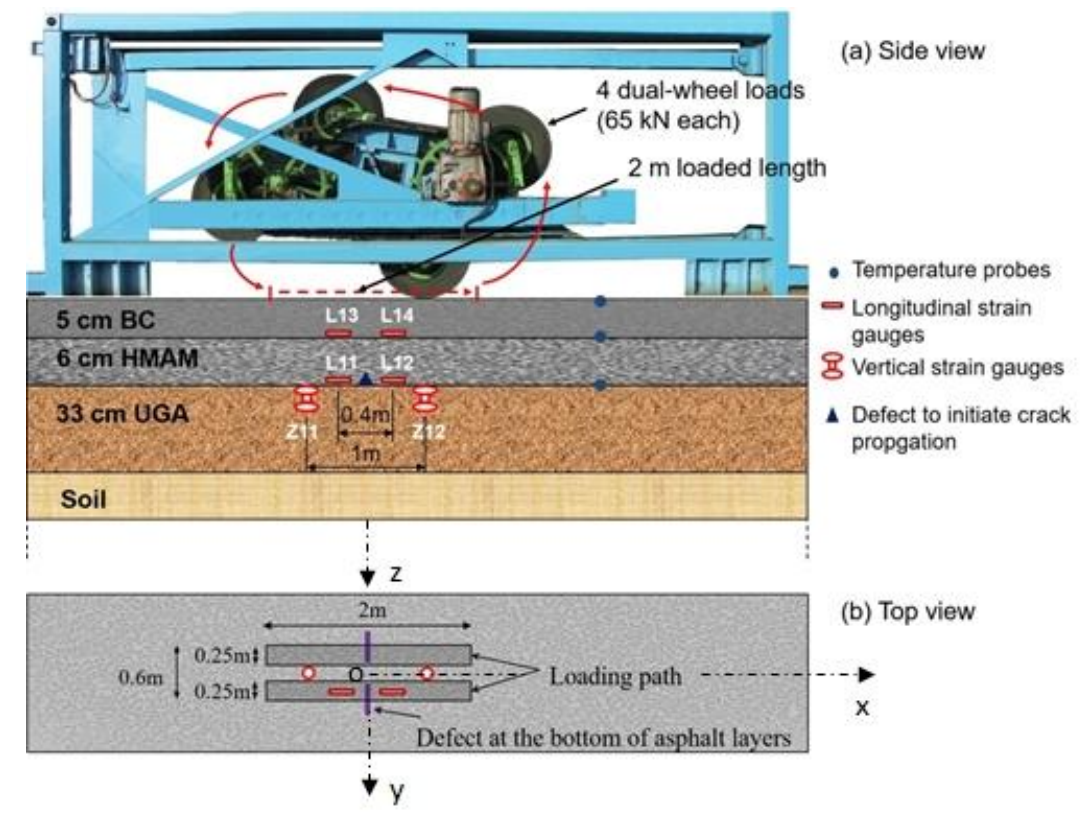

Fig. 1 The FABAC machine and the instrumented pavement (from Nguyen et al. 2019)

\section{Main Facts Observed Between Cycles \#0 and \#350,000}

The measurements obtained between cycles $\# 0$ and $\# 350,000$ are summarized in Figure 2.

- FWD deflection and vertical strains in UGA

Figure $2 \mathrm{a}$ shows the global increase of deflection measured under the FWD plate for the campaigns performed at cycles $\# 0$ and $\# 350,000$. A large increase of the vertical strain amplitudes (Z11 and Z12 gage measurements) in UGA is 
also observed during this FABAC loading stage (Figure 2b). These observations reflect a loss of bearing capacity of the pavement foundation (soil + UGA) which is attributed to water suction induced by a pumping effect resulting from the quasi-uninterrupted repetition of FABAC loads. Moreover, at cycle $\# 350,000$ two local peaks of deflection can be noticed on the FWD profile in $x \approx-0.40 \mathrm{~m}$ and $x \approx 0 \mathrm{~m}$.

- Horizontal strains in the HMAM and BC layers

Figure $2 \mathrm{c}$ and Figure $2 \mathrm{~d}$ show significant changes in the horizontal strains recorded under the passing of the FABAC loads. In particular, a large increase of amplitude for gage L11 and changes in shape of signals L13 and L14 (from contraction to extension) are noticed in the course of loading.

- Visual inspection of the pavement surface

A transverse crack in two pieces popping up at the pavement surface above the metal angles is observed around cycle $\# 1,000,000$.

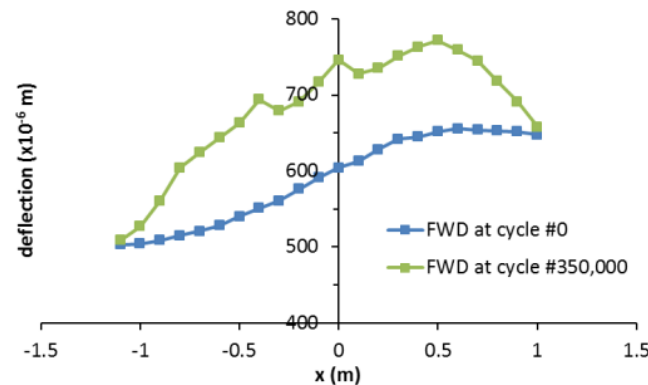

(a)

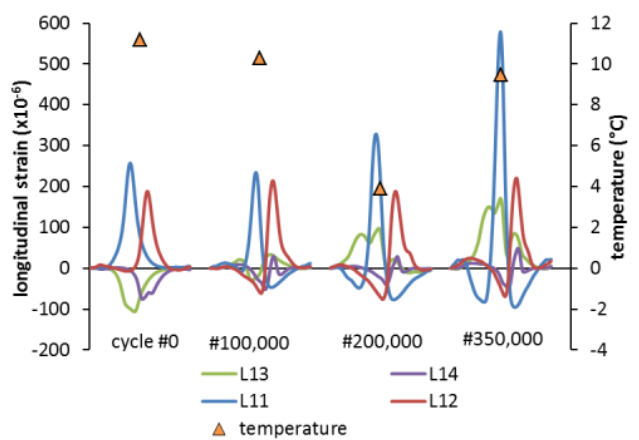

(c)

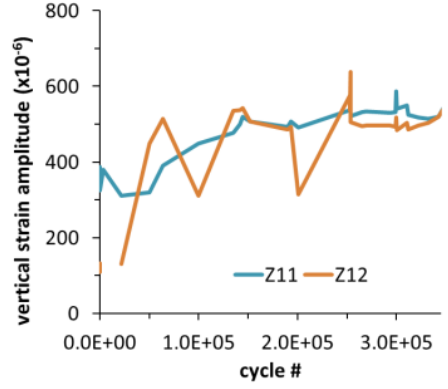

(b)

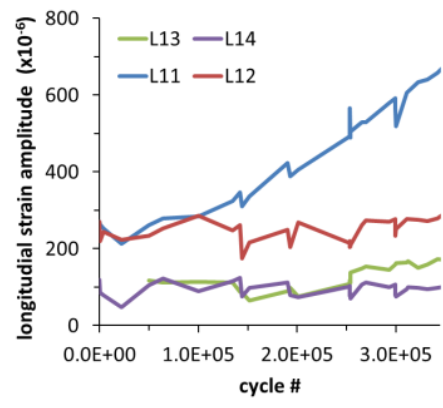

(d)

Fig. 2 Evolution of the measurements between cycle $\# 0$ and $\# 350,000$ (after Nguyen et al. 2019): a) FWD measurements (central geophone), b) vertical strain amplitude in UGA, c) samples of longitudinal strain profiles recorded in the asphalt layers under the passing of a FABAC load, d) evolution of the longitudinal strain amplitude measured in the asphalt layers 


\section{Modeling of the FABAC and FWD Results}

M4-FEM is used to analyze the FWD and FABAC loading results reported above. The ALT pavement is modeled as a 3-layer structure resting on Winkler springs. The two upper layers \#1 and \#2, numbered from top to bottom, represent the BC and HMAM bituminous layers. Layer \#3 represents the upper part of the UGA layer $(15 \mathrm{~cm})$, which includes the vertical strain gauges Z11 and Z12. The Winkler springs are supposed to represent the remaining part of the UGA layer $(15 \mathrm{~cm})$ and soil. Table 1 summarizes the material and geometry data used in M4-FEM. The values of the equivalent elastic modulus $\left(E^{i}\right)$ for the bituminous layers are chosen accordingly to the loading rate of the FWD plate or the FABAC wheels, converted in terms of frequency $(35 \mathrm{~Hz}$ for $\mathrm{FWD} ; 1 \mathrm{~Hz}$ for FABAC loads). Adjustment is done also with regards to the temperature prevailing in the bituminous layers at the time of the measurements. For the sake of simplification, the Young modulus of layer \#3 and the stiffness of the Winkler springs are assumed uniform by considering however two different sets of values to take into account the loss of bearing capacity of the soil foundation between cycles $\# 0$ and $\# 350,000$. The FWD load $(65 \mathrm{kN})$ is modeled as a uniform pressure distribution $(0.72 \mathrm{MPa})$ over a square area $(30 \mathrm{~cm} \times 30 \mathrm{~cm})$ which is moved along the $\mathrm{x}$-axis. The FABAC dual wheel loads are applied on two rectangles $(20 \mathrm{~cm} \times 26 \mathrm{~cm})$ with a center-to-center distance equal to $40 \mathrm{~cm}$ (pressure $=0.63 \mathrm{MPa}$ ). Due to symmetry of the M4-5n mesh used further, only one imprint is considered in the computations.

Table 1 Geometry and material data set for the modeling of the FWD or the FABAC loads

\begin{tabular}{|c|c|c|c|c|c|c|}
\hline \multirow[b]{2}{*}{ Layer i } & \multirow[b]{2}{*}{$\begin{array}{c}\text { Thickness } \\
e^{i}(\mathrm{~m})\end{array}$} & \multirow[b]{2}{*}{$\begin{array}{l}\text { Poisson's } \\
\text { ratio } v^{i}\end{array}$} & \multicolumn{2}{|c|}{ cycle \#0 } & \multicolumn{2}{|c|}{ cycle \#350 000} \\
\hline & & & $\begin{array}{l}E_{F W D}^{i}(M P a) \\
\left(12^{\circ} \mathrm{C}, 35 \mathrm{~Hz}\right)\end{array}$ & $\begin{array}{c}E_{F A B A C}^{i}(M P a) \\
\left(12^{\circ} \mathrm{C}, 1 \mathrm{~Hz}\right)\end{array}$ & $\begin{array}{l}E_{F W D}^{i}(M P a) \\
\left(15^{\circ} \mathrm{C}, 35 \mathrm{~Hz}\right)\end{array}$ & $\begin{array}{c}E_{F A B A C}^{i}(M P a) \\
\left(8^{\circ} \mathrm{C}, 1 \mathrm{~Hz}\right)\end{array}$ \\
\hline $1(\mathrm{BC})$ & 0.05 & 0.35 & 14000 & 8500 & 12500 & 11000 \\
\hline 2 (HMAM) & 0.06 & 0.35 & 19000 & 14000 & 17500 & 16500 \\
\hline 3 (UGA) & 0.15 & 0.35 & \multicolumn{2}{|c|}{420} & \multicolumn{2}{|c|}{350} \\
\hline \multicolumn{3}{|c|}{ Winkler springs $(\mathrm{MPa} / \mathrm{m})$} & \multicolumn{2}{|c|}{60} & \multicolumn{2}{|c|}{50} \\
\hline
\end{tabular}

Several scenarios combining vertical cracks in layer 2 and debonding at the interface between layers 1 and 2 are compared to the main experimental findings. Only the studied scenario that presents the best match with the experimental data is detailed below. 


\subsection{Description of the Selected Damage Scenario}

Figure 3 depicts the scenario of pavement damaging that leads to a good match between the M4-5n simulations and the experimental results (FWD + FABAC) after 350,000 cycles. This scenario includes (i) a loss of the bearing capacity of the UGA layer and soil, (ii) two vertical cracks in layer 2, (iii) and a debonding area at the interface between the two asphalt layers. One of the cracks (denoted $C 1$ ) is assumed to have developed vertically in plane $O y z$ right above the metal angles in layer 2 (Figure 1), as expected from the experimental setup. The other crack $(C 2)$, not planned initially, crosses gage L11 but is believed to have propagated not fully parallel to plane $y z$. The debonding area $(D)$ is assumed to have initiated from the $C 1$ crack tip to further develop to the right towards gage L14. One can notice that this scenario is unsymmetrical with regards to the $O y z$ mid-plane of the test section. $C 2$ also induces a priori a kind of non-symmetric pattern with regards to the $O x z$ mid-plane.

This scenario was actually deduced from M4-5n simulations performed with the following simplified assumptions. The loss of bearing capacity of the pavement foundation is taken into account by decreasing the Young modulus of the UGA layer and the stiffness of the Winkler springs (Table 1). The two cracks are modeled as vertical areas in $y z$-planes extending over the whole thickness and width of layer 2 . The a priori oblique path of $C 2$ is taken into account in a simplified way by positioning the $y z$-plane related to $C 2$ in $x=-0.2 m$ when modeling the FABAC test and in $x=-0.4 m$ for the FWD loading. Besides, $D$ is represented by a rectangle also extending over the whole width of the pavement and from $x=0$ to $0.3 \mathrm{~m}$.

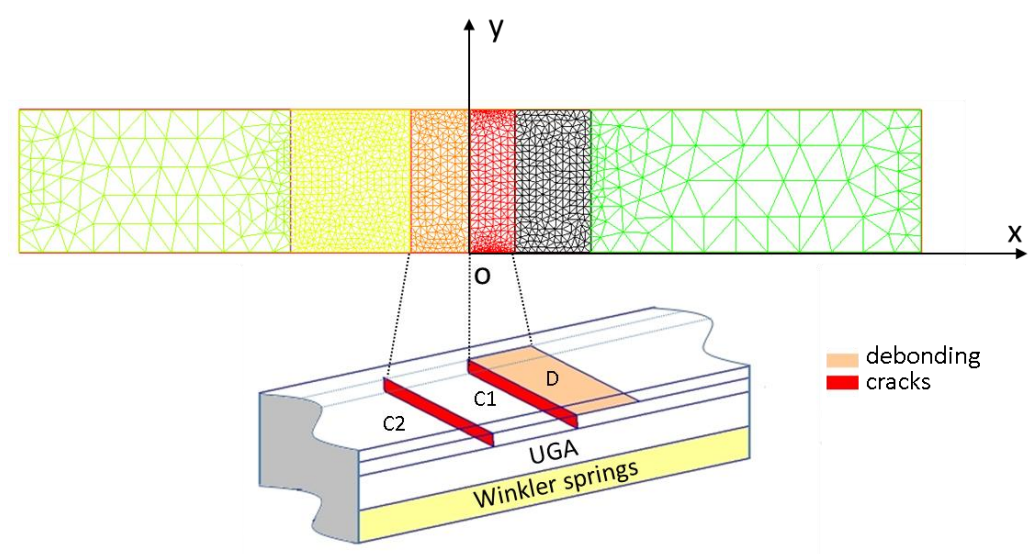

Fig. 3 3D sketch of the damage scenario at cycle $\# 350,000$ and the related 2D M4-5n mesh (bottom view) 
The following sections show the comparison between the experimental results and the M4-5n simulations that led to the proposal of this scenario among many others studied.

\subsection{Comparison between experimental and M4-5n results for the FWD test campaigns}

M4-FEM is first used to simulate the FWD campaigns performed at cycles \#0 and $\# 350,000$. The numerical value of deflection at cycle $\# 0$ is found equal to $620 \times$ $10^{-6} \mathrm{~m}$ which is close to the mean experimental value (Figure $2 \mathrm{a}$ ). Figure $4 \mathrm{a}$ shows the numerical and test results plotted as the difference between the values at cycles \#350,000 and \#0. A good match is obtained in the circulated area except for the end points from which the deflection tends to a plateau value around $80 \times$ $10^{-6} \mathrm{~m}$ corresponding to the difference in deflection calculated with the two parameter sets of the UGA layer and the Winkler springs. By using the initial set of values outside the circulated area in the simulation at 350,000 cycles this difference would actually vanish, confirming that the decrease of the bearing capacity is localized under the FABAC loading (water pumping effect). The simulation results well account for the three peaks observed in the central region of the ALT pavement section. The two first peaks are due to the presence of cracks in $x=0$ and $x=-0.4 \mathrm{~m}$ whereas debonding is responsible for the third one. Note that the numerical curve results from the combination of those of Figure $4 \mathrm{~b}$ obtained considering $D$ as fully bonded or debonded.

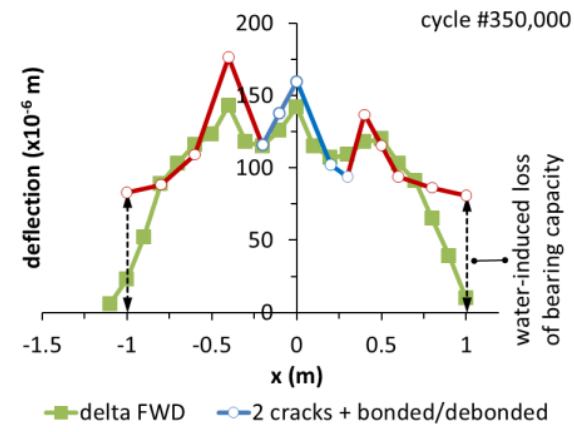

(a)

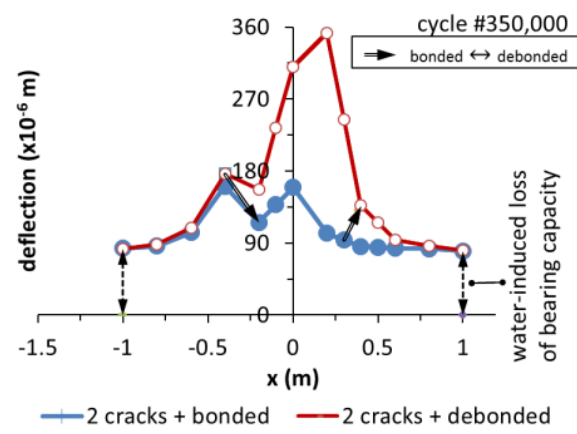

(b)

Fig. 4 Difference in FWD deflection between cycle \#0 and \#350,000. (a) Comparison between the measurements and the M4-5n simulation, (b) M4-5n results for fully bonded or debonded behavior of $D$

In Figure $4 \mathrm{a}$ the bonded condition is considered when the center of the FWD loading plate is located between $\mathrm{x}=-0.2$ and $\mathrm{x}=0.3 \mathrm{~m}$, i.e. in a region 
encompassing closely that above the geometrical area $D$. This region is also that for which interpenetration is found at the $D$ location in case of debonding. It seems then logical to switch to the assumption of bonding for these load locations.

\subsection{Comparison between experimental and M4-5n results for the FABAC loads}

Figure 5 shows the comparison between the strain gage measurements and the corresponding computed values in layers 1 and 2 at cycle \#0. The abscissa indicates the location of the FABAC wheels. A fair agreement is obtained showing contraction at bottom of layer 1 and extension at the bottom of layer 2 .

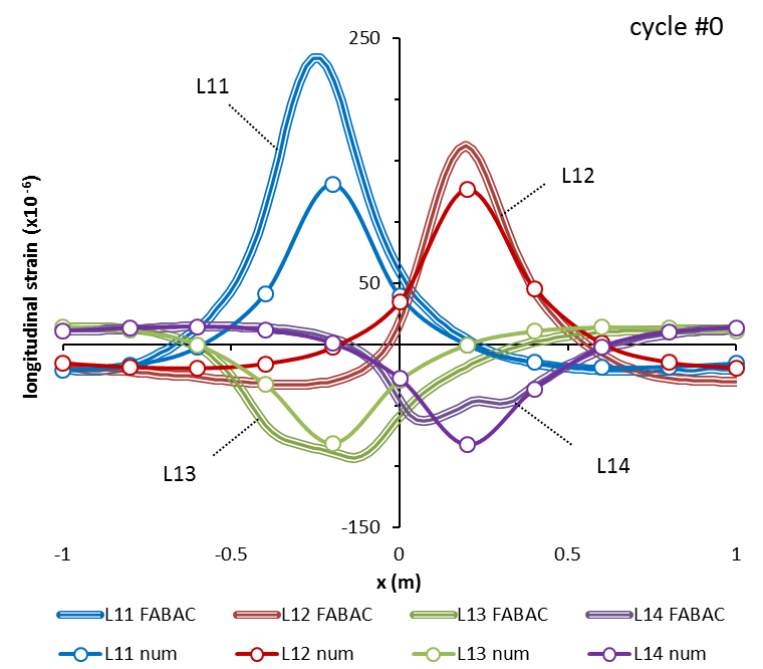

Fig. 5 Comparison between the longitudinal strains measured and computed at cycle \#0

Figure 6 shows the same comparison but at cycle \#350,000 considering in the simulation the damage scenario mentioned earlier (Figure 3). In the computations, debonding is assumed in $D$ excepted when the loads are moved right above this area where in that case full bonding is assumed between the 2 layers. The change in the contact condition for $D$ is taken into account approximately in the computation of the strain response $\varepsilon_{L}$, at the location of the strain gages, under the moving loads, using the incremental equations Eq. 1 and Eq. 2:

$\varepsilon_{L}^{b}\left(x_{\text {load }}\right)=\varepsilon_{L}^{d}\left(x_{d / b}\right)+\varepsilon_{L}^{b}\left(x_{\text {load }}\right)-\varepsilon_{L}^{b}\left(x_{d / b}\right)$
$\varepsilon_{L}^{d}\left(x_{\text {load }}\right)=\varepsilon_{L}^{b}\left(x_{b / d}\right)+\varepsilon_{L}^{d}\left(x_{\text {load }}\right)-\varepsilon_{L}^{d}\left(x_{b / d}\right)$ 
in which superscripts $b, d$ denote the bonded and debonded conditions, respectively. $x_{b / d}$ and $x_{d / b}$ are the load locations at which the contact condition is assumed to change from bonding to debonding and vice versa.

Besides, since L11 is crossed by a crack, a pseudo-strain is considered for this gage. This pseudo-strain is computed as the difference in the horizontal displacement between both ends of the gage over its length.

Globally, a relatively good agreement is obtained between the change in longitudinal strains observed experimentally between cycles \#0 and \#350,000 and that computed by M4-5n. In particular, the large evolution recorded by L11 between cycles \#0 and \#350,000 is well described. The change from contraction to extension for gages L13 and L14 is also well captured numerically by the damage scenario considered here. Note however that the peak of tensile strain recorded by L12 which remains almost unchanged in the measurement rather diminishes in the simulation. In addition, the numerical curve for L12 at cycle \#350,000 does not exhibit a peak of contraction as does the experimental curve prior to the arrival of the load right above this gage.
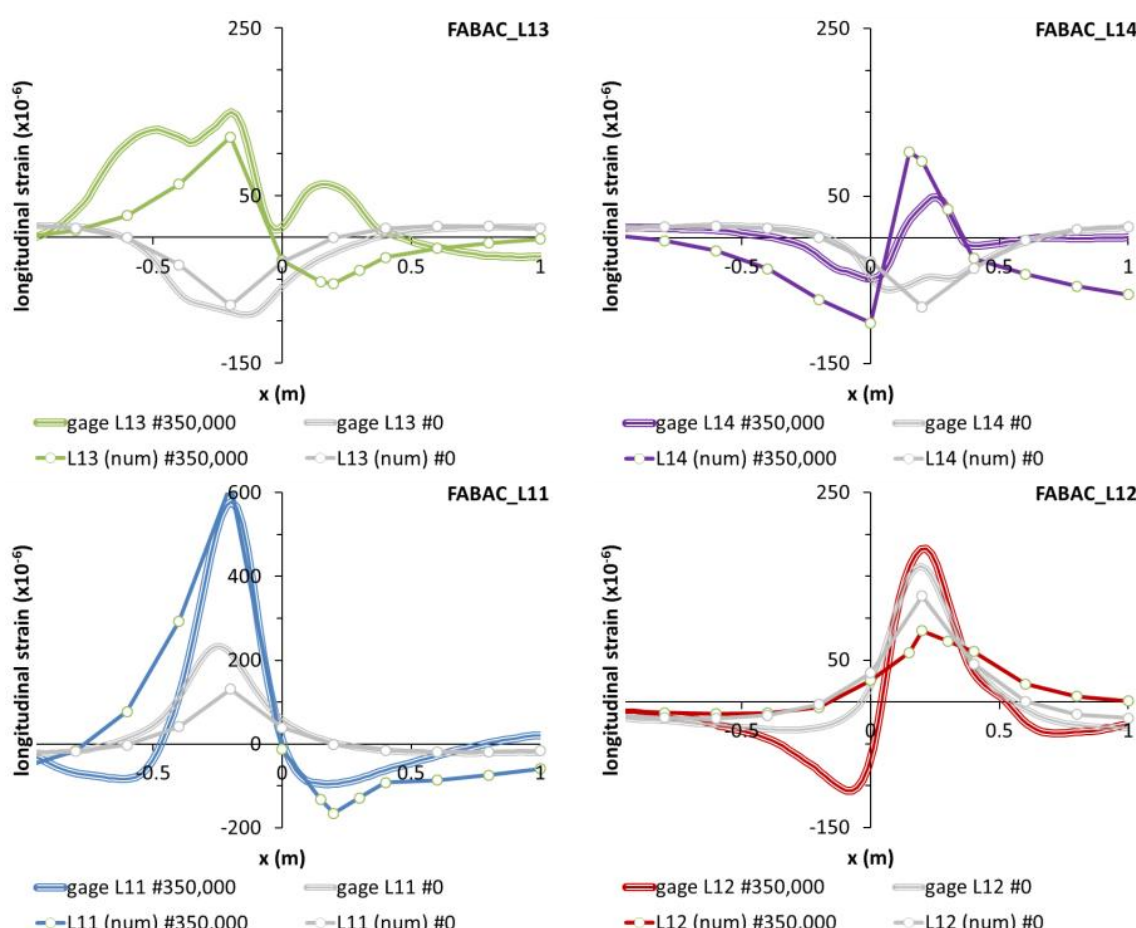

Fig. 6 Comparison between the longitudinal strains measured and computed by M4-5n at cycle \#350,000 (the grey curves recall the comparison at cycle \#0)

The scenario considered here is probably not fully representative of the reality even though it allows us to reflect the main features resulting from damaging of 
the pavement. An appropriate and careful deconstruction of the test section should help validate and specify the actual cracking/debonding pattern.

\section{Conclusion}

The M4-5n associated to a Winkler foundation was applied to the numerical analysis of a FABAC ALT test performed on a pavement composed of two bituminous layers at the bottom of which a flaw was placed on purpose to initiate and localize crack growth. The mechanical response of the pavement was computed for many different damage scenarios taking advantage of the versatility of M4-FEM to model the geometry of cracking/debonding patterns. One of these scenarios which led to a satisfactory match with the set of test measurements was presented in this paper. The future development and implementation of fatigue criteria will help the selection of scenarios and should also lead to a better understanding of the fracture mechanisms at play.

\section{References}

Aunis J, Balay J-M (1998). An applied research programme on continuous reinforced concrete pavements: the FABAC project. 8th Int. Symp. on Concrete Roads, Lisbon, 13-16 Sept. 1998.

Buttlar WG, Chabot A, Dave EV, Petit C, Tebaldi G (2018). Mechanisms of Cracking and Debonding in Asphalt and Composite Pavements - RILEM State-of-the-Art Report Series, Vol. 28, Springer International Publishing. doi: 10.1007/978-3-319-76849-6

Chabot A (1997). Analyse des efforts à l'interface entre les couches des matériaux composites à l'aide de Modélisations Multiparticulaires des Matériaux Multicouches (M4). PhD thesis, ENPC, Paris. Available on https://tel.archives-ouvertes.fr/tel-00197853/

Chabot A, Tran QD, Ehrlacher A (2007). A modeling to understand where a vertical crack can propagate in pavements. Proc. of the Int. Conf. on Advanced Characterization of Pavement and Soil Engineering Materials (CRC Press) 1: 431-440, Athens, 20-22 June 2007.

Chabot A, Pouteau B, Balay J-M, De Larrard F (2008). FABAC Accelerated Loading Test of Bond between Cement Overlay and Asphalt layers. Proceedings (CRC Press) of the 6th Int. RILEM Conf. Cracking in Pavements, 16-18 June, Chicago, 671-681.

Hecht F (2012). New development in FreeFem++. Journal of Numerical Mathematics 20 (3-4), 251-265. 65Y15. doi 10.1515/jnum-2012-0013

Nasser H, Chabot A (2018). A Half-analytical Elastic Solution for 2D Analysis of Cracked Pavements. Advances in Engineering Software 17: 107-122. doi: 10.1016/j.advengsoft.2017.06.008

Nasser H, Chupin O, Piau JM, Chabot A (2018). Mixed FEM for solving a plate type model intended for analysis of pavements with discontinuities. Road Materials and Pavement Design 19 (3): 496-510. doi: 10.1080/14680629.2018.1418653

Nguyen ML, Chupin O, Blanc J, Piau JM, Hornych P, Lefeuvre Y (2019). Investigation of crack propagation in asphalt pavement based on APT results and LEFM analysis. ASTM Journal of Testing and Evaluation 48. doi: 10.1520/JTE20180933 